\title{
The uniaxial tensile deformation of Ni nanowire: atomic-scale computer simulations
}

\author{
Yu-Hua Wen ${ }^{\mathrm{a}}$, Zi-Zhong Zhu ${ }^{\mathrm{a}, *}$, Gui-Fang Shao ${ }^{\mathrm{b}}$, Ru-Zeng Zhu ${ }^{\mathrm{c}}$ \\ ${ }^{\mathrm{a}}$ Department of Physics, Xiamen University, Xiamen 361005, PR China \\ ${ }^{\mathrm{b}}$ Department of Computer Science, Chongqing Institute of Technology, Chongqing 400050, PR China \\ ${ }^{\mathrm{c}}$ LNM, Institute of Mechanics, Chinese Academy of Sciences, Beijing 100080, PR China
}

Received 13 September 2004; accepted 25 October 2004

Available online 18 December 2004

\begin{abstract}
The mechanical deformations of nickel nanowire subjected to uniaxial tensile strain at $300 \mathrm{~K}$ are simulated by using molecular dynamics with the quantum corrected Sutten-Chen many-body force field. We have used common neighbor analysis method to investigate the structural evolution of $\mathrm{Ni}$ nanowire during the elongation process. For the strain rate of $0.1 \% / \mathrm{ps}$, the elastic limit is up to about $11 \%$ strain with the yield stress of $8.6 \mathrm{GPa}$. At the elastic stage, the deformation is carried mainly through the uniform elongation of the distances between the layers (perpendicular to the $Z$-axis) while the atomic structure remains basically unchanged. With further strain, the slips in the $\left\{\begin{array}{llll}1 & 1 & 1\end{array}\right\}$ planes start to take place in order to accommodate the applied strain to carry the deformation partially, and subsequently the neck forms. The atomic rearrangements in the neck region result in a zigzag change in the stress-strain curve; the atomic structures beyond the region, however, have no significant changes. With the strain close to the point of the breaking, we observe the formation of a one-atom thick necklace in Ni nanowire. The strain rates have no significant effect on the deformation mechanism, but have some influence on the yield stress, the elastic limit, and the fracture strain of the nanowire.
\end{abstract}

(C) 2004 Elsevier B.V. All rights reserved.

PACS: 61.46. +w; 62.20.Fe; 87.64.Aa

Keywords: Nanowires; Mechanical deformation; Molecular dynamics

\section{Introduction}

Exhibiting unique mechanical, electronic, optical, and magnetic properties, materials based on

\footnotetext{
*Corresponding author. Fax: 865922189426.

E-mail address: zzhu@xmu.edu.cn (Z.-Z.Zhu).
}

nanometer-sized structures have attracted a great deal of interests in the past two decades, opening up a range of new applications [1-3]. As one of the most important one-dimensional (1D) nanometer materials, the metal nanowires have been expected to play an important role in future electronic, optical and nanoelectromechanical devices [4-9]. 
The study of mechanical behavior of nanoscale metallic wires is becoming an increasingly important area of materials science, because it is not only of scientific interests but also has implications for constructing materials with specific mechanical properties, for instance, as reinforcing fibers in high-strength/light-weight composite materials [10]. However, measuring the mechanical and electrical properties of a nanowire is a difficult task due to the small dimensions [11]. In recent years, the deformation of nanowires have been studied by molecular dynamics simulations using either embodied-atom-method (EAM) [12-15] or effective-medium theory (EMT) $[16,17]$ potentials as well as first-principles method based on the density functional theory (DFT) [18-21]. These studies focus on the correlation of the force (associated with the changes in the bonding of the nanowire) and the conductance for the metallic $\mathrm{Au}, \mathrm{Ni}$, and $\mathrm{Na}$ nano-contacts $[17,22]$ and $\mathrm{Au}, \mathrm{Ni}, \mathrm{Al}, \mathrm{Na}$, and $\mathrm{SiSe}_{2}$ nanowire [12,16,20,23]. The structural transformation takes place in nanoscale metallic wires under uniaxial strain [12,14,17-18,23]. At lower strain rates, the $\mathrm{Ni}$ nanowire shows superplasticity [12,13], and at sufficiently high strain rates, it can transform continuously to an amorphous metal at constant temperature [13,14,23]. The Au nanowires, before breaking under tensile stress, can get as thin as one-atom chains, and as long as five suspended atoms [18,21]. Sorensen et al. [17] have simulated the mechanical deformation of atomic-scale metallic contacts under tensile strain, and found that various defects such as intersecting stacking faults, local disorder, and vacancies were created during the deformation. There are many unanswered questions relating to the mechanical mechanism, especially those related to the tensile process, and are expected to be illustrated in future.

Since metal nanowires have a number of exciting potential applications in nanoscale electronic devices, it is necessary to develop a quantitative understanding of the mechanical and structural properties of such metal nanowires. Molecular dynamics (MD) simulations provide a useful tool at the atomic level to analyze the structural, mechanical, and thermodynamic properties of nanomaterials, and eventually to design materials with desired characteristics. In the present work, we have used MD to simulate the uniaxial tensile deformation of $\mathrm{Ni}$ nanowire, and investigate the structural evolution of the mechanical deformation process. Details of the MD simulation are presented in the following section; we arrange the brief discussion of the MD results and comparison with other results in the third section. The main conclusions are summarized in the fourth section.

\section{Simulation process}

In the present paper, MD simulations have been performed with the quantum corrected SuttenChen (Q-SC) type many-body force field modified by Kimura et al. [24] in which the parameters were optimized to describe the lattice parameter, cohesive energy, bulk modulus, elastic constants, phonon dispersion, vacancy formation energy, and surface energy, leading to an accurate description of many properties of metals and their alloys [25-27]. We have simulated the tensile process of $\mathrm{Ni}$ nanowire at room temperature. The initial geometry of $\mathrm{Ni}$ nanowire has been constructed from a large cubic FCC single crystal of nickel using certain cylindrical cutoff radii centered at a cubic interstitial site, in which the crystallographic orientations in the $X-, Y$ - and $Z$-axis are taken to be in the direction of [1 000$],$ [ $\left.\begin{array}{lll}0 & 1 & 0\end{array}\right]$, and [ $\left.\begin{array}{lll}0 & 0 & 1\end{array}\right]$, respectively. In the $X$ and $Y$ directions, the $\mathrm{Ni}$ nanowire constructs a finite number of unit cells, while in the $Z$-direction an infinite wire was obtained by applying the periodic boundary conditions. The diameter of the nanowire is $2.96 \mathrm{~nm}$ (8.4 FCC unit cells), and the length of the nanowire adopt is initially $14.1 \mathrm{~nm}$ (about 40 FCC unit cells in $z$-direction). First, the $\mathrm{Ni}$ nanowire is relaxed by running $50 \mathrm{ps}$ at $300 \mathrm{~K}$ in order to eliminate the unfavorable configurations. Subsequently, the uniaxial deformation of the nanowire is simulated at constant temperature and constant strain rate in which the length (in the $Z$-direction) of the nanowire is increased gradually. Three different strain rates, $0.25 \% / \mathrm{ps}, 0.1 \%$ / ps, and $0.025 \% / \mathrm{ps}$, are applied on the nanowire to study the deformation mechanism. It should be 
emphasized that the loading process of the tensile strain is different from those described in Refs. $[13,14]$, where the tensile strain with increments of $0.5 \%$ in its total length after some time steps. In the present work, the tensile strain is increased by $5 \times 10^{-6}, 2 \times 10^{-6}, 5 \times 10^{-7}$ in each time step along the $Z$-direction for the strain rate of $0.25 \% / \mathrm{ps}, 0.1 \% / \mathrm{ps}$, and $0.025 \% / \mathrm{ps}$, respectively. During the deformation process, the temperature is kept at $300 \mathrm{~K}$ based on the NoseHoover chain dynamics [28], and the diameter of the nanowire is allowed to shrink (or expand) in order to keep the transversal component of the stress $\left(\sigma_{x x}\right.$ and $\left.\sigma_{y y}\right)$ close to zero based on the Berendsen approach [29], while the stress component $\sigma_{z z}$ is calculated at the corresponding strain. In MD simulations, the amplitudes of initial velocities of all atoms are chosen according to the Maxwell distribution. The initial directions of the velocities are distributed randomly so that the velocity averaged over all atoms is zero and remains zero for all the time steps. All the atomic trajectories were followed by integrating Newton's equation of motion for each atom with the LeapFrog algorithm [30]. The time step in the simulation was $2.0 \times 10^{-15} \mathrm{~s}$, the unit of energy was in $\mathrm{eV}$, and the unit of length in $a_{0}=3.524 \AA$ which is the lattice parameter of bulk $\mathrm{Ni}$ at room temperature.

In MD simulations, we can obtain positions, velocities, and accelerations for all the atoms in the system at each time step. According to these data, the information of the structural evolution may be acquired through the common neighbor analysis (CNA) [31] method. Using this method, we have calculated CNA indices for a number of atomic configurations extracted from the MD simulations. The different types of pairs are associated with different types of local order. All bonded pairs in the FCC crystal are of type 1421 , while the HCP crystal has equal numbers of type 1421 and 1422 . Considering the initial FCC order of $\mathrm{Ni}$ nanowire, here we define only three categories of atoms: 'FCC' atoms, having a local FCC order and considered to be inside the nanowires; 'HCP' atoms, having a local HCP order and classified as stacking faults; and 'other' atoms, all the other atoms considered as belonging to surface. The
CNA method has already been used successfully to analyse the structural evolution during the deformation and melting process [32-34].

\section{Results and discussion}

Firstly, we present the simulated results at the strain rate of $0.1 \% / \mathrm{ps}$. Fig. 1 shows the stressstrain curve of the Ni nanowire at $300 \mathrm{~K}$. The stress increases linearly with the strain up to $8.62 \mathrm{GPa}$ (at $11.17 \%$ strain). The elastic limit is about $11 \%$, close to the value of $10.5 \%$ simulated by Branicio and Rino [13]. With the further tensile, the stress drops abruptly to $2.42 \mathrm{GPa}$ (at $11.89 \%$ strain) and the stress-strain relations display a zigzag curve as the strain is increased. When the strain reaches $39.32 \%$, the stress is close to zero, which means a breaking of the nanowire. According to the stress-strain curves under the elastic range, we obtain the Young's modulus $E_{\left[\begin{array}{ll}0 & 1\end{array}\right] \text { as }}$ $80.6 \mathrm{GPa}$ along the $\left[\begin{array}{lll}0 & 0 & 1\end{array}\right]$ direction, which is appreciably lower than that of the nanowire (about $90 \mathrm{GPa}$ ) calculated by Branicio and Rino [13]. This difference may have resulted from two aspects: the diameter of the nanowire and the potential used in the MD simulations. The yield stress of $8.62 \mathrm{Gpa}$, obtained from the stress-strain curve, is far above that of experimental bulk Ni

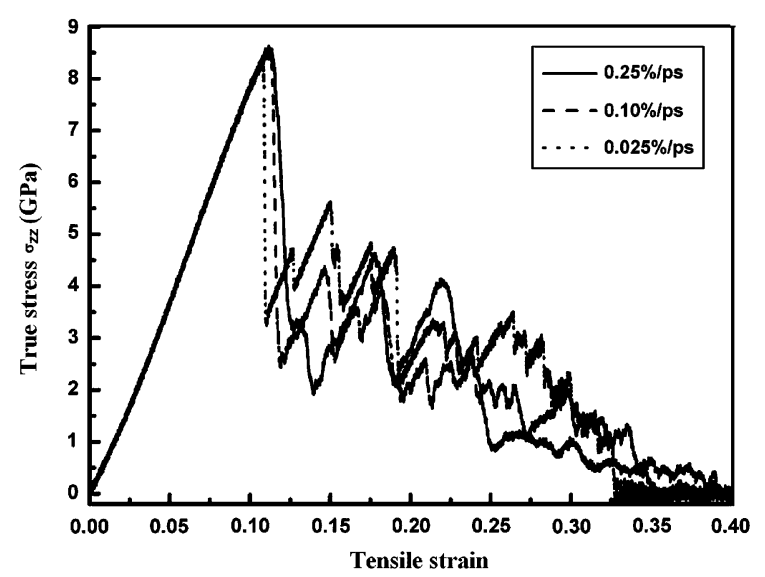

Fig. 1. The normal stress along $Z$-direction $\sigma_{z z}$ as a function of the tensile strain for Ni nanowire at $300 \mathrm{~K}$. The strain rates are $0.25 \% / \mathrm{ps}, 0.1 \% / \mathrm{ps}$, and $0.025 \% / \mathrm{ps}$, respectively. 
(about $0.14 \mathrm{GPa}$ ). When comparing the simulation results of yield stress to the experimental one caution is required since the experimental bulk samples have defects, such as dislocations, voids, and impurities which are advantageous for the deformation process. The nanowire simulated in present paper, however, is defect-free and pure. On the other hand, the yield stress increases with the decreasing grain size, which can be expressed by the well-known Hall-Petch relation [35,36]. In nanoscale regime, this relation has been confirmed by experimental data [37-39]. According to the Hall-Petch relation, nanocrystalline materials would display great yield stress as the grain size is reduced to nanometer dimension. The recent paper reports that the experimental value of the yield stress is $2.25 \mathrm{GPa}$ for nanocrystalline $\mathrm{Ni}$ with a mean grain size of $26 \mathrm{~nm}$ [40]. This value, about one-third of the present simulated result, is reasonable considering that nanocrystalline $\mathrm{Ni}$ has a large fraction of grain boundaries and defects.

Fig. 1 also shows the strain rates effects on the stress-strain curves. For all the strain rates employed, the stresses increase linearly with the strain when the strain is below $11 \%$. Below the elastic limit, the stress-strain curves for the three strain rates are almost completely overlapped. This implies that the strain rate has no significant effect on the elastic properties of Ni nanowire. For the strain of $0.25 \% / \mathrm{ps}$, the first peak of the stress, i.e. the yield stress, is $8.68 \mathrm{GPa}$ at the strain of $11.36 \%$; for the strain rate of $0.10 \% / \mathrm{ps}$ and $0.025 \% / \mathrm{ps}$, the yield stress is $8.62 \mathrm{GPa}$ (at $11.17 \%$ strain) and $8.42 \mathrm{GPa}$ (at $10.76 \%$ strain), respectively. Obviously, the lower strain rate results in the lower yield stress, but the change of the yield stress is remarkably small. Once the strain exceeds the yield point, the stress drops rapidly to about $3.0 \mathrm{GPa}$, and then rises in a repeating increase/decrease cycle with the further applied strain. The strain, in which the $\mathrm{Ni}$ nanowire is failure, is different for the three strain rates. The nanowire breaks at the strain of $32.76 \%$ for the $0.025 \% / \mathrm{ps}$ strain rate, but at the strain of $40.42 \%$ for the $0.25 \% / \mathrm{ps}$ strain rate. Therefore, the strain rate has some influence on the fracture of the nanowire.
In order to understand how the stress varies with the strain, we have studied the structure evolution of the tensile process under the strain rate of $0.1 \% / \mathrm{ps}$ using CNA methods. Considering that pairs beside types of 1421 and 1422 do not reveal any useful information for Ni FCC nanowire, we have classified all atoms into three categories: 'FCC' atoms, 'HCP' atom, and 'other' atoms, as mentioned previously. Fig. 2 presents the tensile strain dependence of the fraction of the three categories of atoms. Comparing Fig. 2 with Fig. 1, we can see that the fraction of all categories of atoms change with the fluctuating stress. When the strain is below $7.5 \%$, the fraction of ' $\mathrm{FCC}$ ' atoms and 'other' atoms keep at $71 \%$ and $29 \%$ with no 'HCP' atoms present in the nanowire, which means that the structure has no change. When the strain is increased from $8.5 \%$ to $11.2 \%$, an obvious decrease of FCC atoms from $70.5 \%$ to $67 \%$ is found, and a corresponding increase of 'other' atoms arise from $29.5 \%$ to $33 \%$, but there is still no 'HCP' atoms found in the system. In this region of strain, some 'FCC' atoms near the surface change into 'other' atoms through atomic rearrangement, which is partially responsible for the deformation. Below $11.2 \%$ strain, the deformation is accommodated mainly through the homogeneous elongation of distances between the layers (perpendicular to the $Z$-axis) with atomic structure remaining basically unchanged. So the elastic limit is about $11.2 \%$, which can also be seen from the approximately linear relationship in stress-strain curve displayed in Fig. 1.

With further strain, the fraction of 'other' atoms increases sharply, up to $38.45 \%$ at strain of $11.4 \%$, and then decreases abruptly to $33.5 \%$ at $12.12 \%$ strain, as shown in Fig. 2(c); meanwhile, the HCP atoms appear at strain of $11.24 \%$ and reach $7.59 \%$ at strain of $11.8 \%$. Comparing Figs. 2(a)-(c), it shows that the 'HCP' atoms come from the rearrangement of 'FCC' atoms in the nanowire, and some of the 'FCC' atoms change into 'other' atoms.

To visualize the process of atomic rearrangements, we display atomic configurations with two different strains, i.e. $11.2 \%$ and $11.8 \%$ in Fig. 3 . At $11.2 \%$ strain, the nanowire has no 'HCP' atom. All ' $F C C$ ' atoms are distributed in the interior 


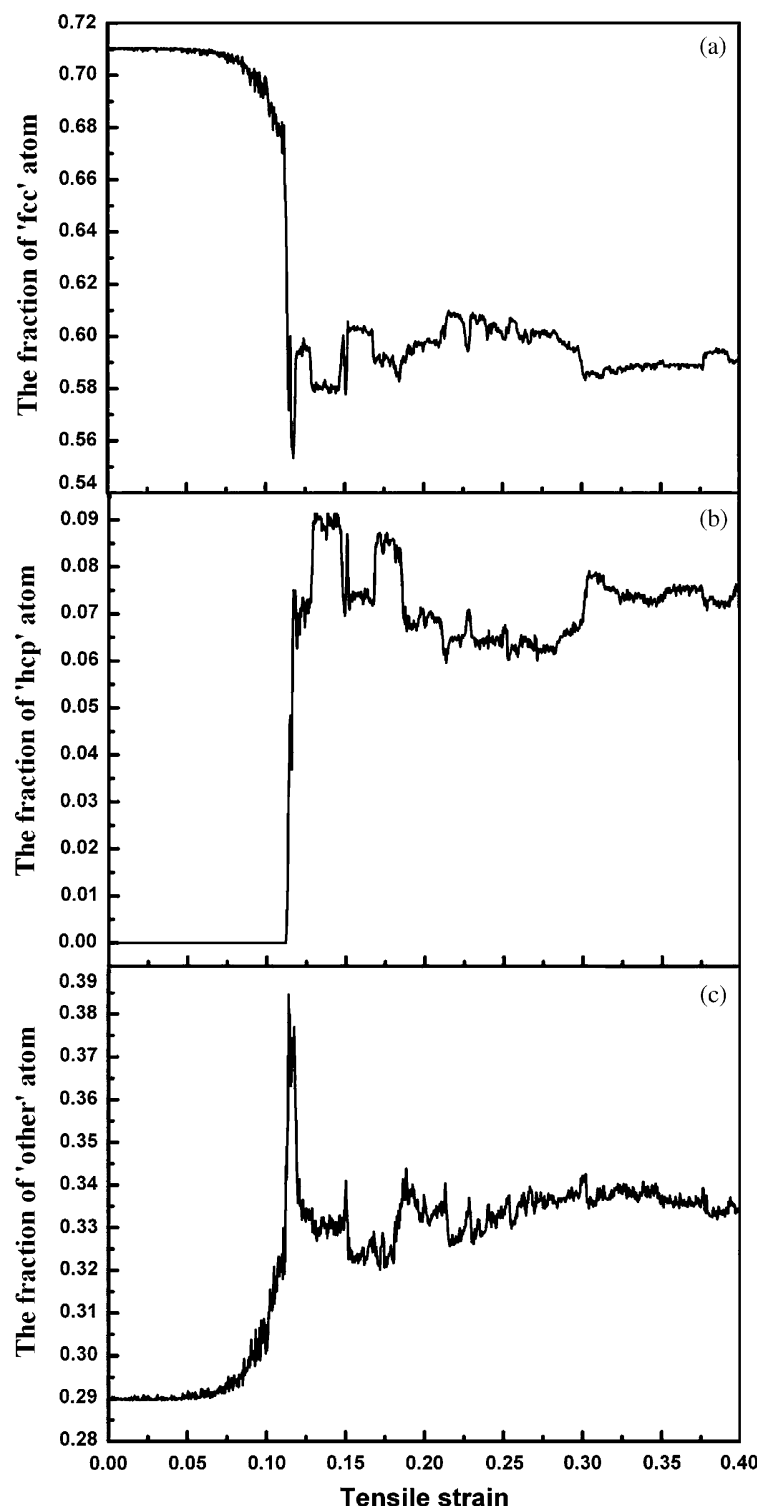

Fig. 2. CNA results for Ni nanowire. The fractions of three categories of atoms are shown as a function of the tensile strain. (a) The fraction of 'FCC' atoms. (b) The fraction of 'HCP' atoms. (c) The fraction of 'other' atoms.

region as shown in Fig. 3(b), and all 'other' atoms are located in the surface layer. The shape of the nanowire keeps its initial column (see Fig. 3(a)). When the strain reaches $11.8 \%$, a large numbers of 'HCP' atoms appearing in the interior, displayed in Fig. 3(c), and the neck has been formed as

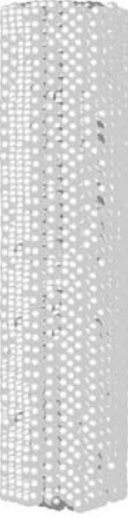

(a)

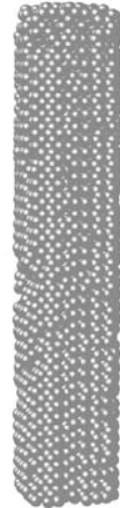

(b)

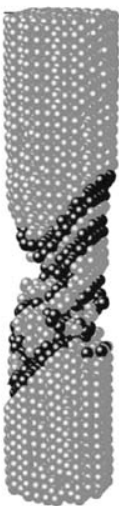

(c)

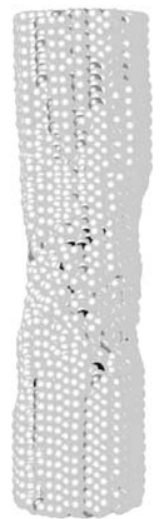

(d)
Fig. 3. Snapshots of atomic configurations of Ni nanowire at $300 \mathrm{~K}$ at the strain rate of $0.1 \% / \mathrm{ps}$. Atoms are color-coded according to the local crystalline order as determined by CNA: gray atoms are in a FCC environment, black atoms are in a local HCP environment which are at stacking faults. Atoms in any other environment are colored light gray which are typically at surface layers. (a) All atoms in $\mathrm{Ni}$ nanowire displayed at $11.2 \%$ strain. (b) Only 'FCC' atoms displayed at $11.2 \%$ strain. (c) Only 'FCC' and 'HCP' atoms displayed at $11.8 \%$ strain. (d) All atoms displayed at $11.8 \%$ strain. Comparing (a) and (b), it can be seen that 'other' atoms are typically in surface layers, and the nanowire keeps its initial column at the strain of $11.2 \%$. We clearly see stacking faults left behind by the slips in the $\left\{\begin{array}{llll}1 & 1 & 1\end{array}\right\}$ planes, and some 'other' atoms and voids appear in the interior at $11.8 \%$ strain.

shown in Fig. 3(d). The appearance of 'HCP' atoms in the system implies the formation of stacking faults caused by the slips in the $\left\{\begin{array}{lll}1 & 1 & 1\end{array}\right\}$ planes. Beyond the elastic limit (above 11.2\% strain), the nanowire cannot keep the prefect FCC structure; plastic deformation starts to take place in order to accommodate the applied strain. The slips, which happened in the $\left\{\begin{array}{lll}1 & 1 & 1\end{array}\right\}$ planes, can partially carry the plastic deformation. In the crystalline structure, the $\left\{\begin{array}{lll}1 & 1 & 1\end{array}\right\}$ planes include four different planes, there are mainly two $\left\{\begin{array}{lll}1 & 1 & 1\end{array}\right\}$ planes playing a role in the present simulations. Some 'other' atoms changed from 'FCC' atoms, occur in the neck region, and some defects, such as voids, are also found in the region.

By monitoring the structure evolution of the nanowire during the tensile process, we display in Fig. 4 the dependence of the diameter of the nanowire on the strain. The calculation method of 


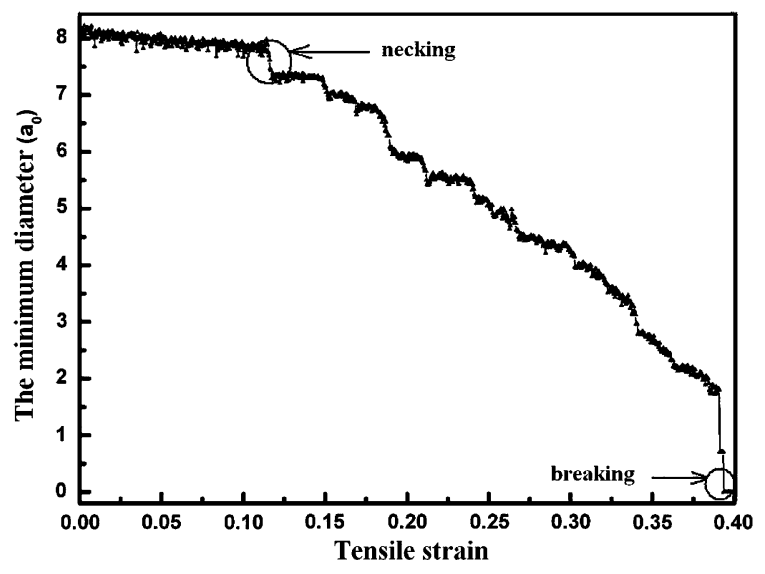

Fig. 4. Dependence of diameter on strain for the Ni nanowire. The diameter is 8.4 under no strain. In order to find when and where the necking and breaking happens, we calculate the minimum of the diameter along the length of the nanowire, and display the minimum value at the different strain. The neck finally formed at $11.8 \%$ strain as shown by the circle. The breaking happens at $39.40 \%$ strain when the minimum of the diameter is zero.

the diameter is the same as in Ref. [41]. It should be mentioned that the diameter is the minimal diameter along the length of the nanowire at the given strain. Below the elastic limit (11.2\% strain), the diameter decreases linearly with the increase of strain. When the strain reaches $11.48 \%$, the diameter begins to exhibit an abrupt decrease; the significant decrease of diameter continues to strain of $11.8 \%$. The reason for the significant decrease of diameter is due to the formation of neck. At $11.48 \%$ strain, the fraction of 'HCP' atoms is $4.83 \%$, which means that the slips in the $\left\{\begin{array}{lll}1 & 1 & 1\end{array}\right\}$ planes happened and these 'HCP' atoms are located in the slipping planes. When the neck forms finally after the slips in the $\left\{\begin{array}{lll}1 & 1 & 1\end{array}\right\}$ planes, the ability of loading on the nanowire decreases sharply, similar to what happens in bulk materials [42].

We also calculate the diameter along the length of the nanowire at each time step for the strain rate of $0.1 \% / \mathrm{ps}$. Before the tensile strain, the diameter along the length of the nanowire fluctuates between 8.15 and 8.74 , and its mean value is 8.42; the distribution of the diameter along the axis of the nanowire is uniform. For the $11.8 \%$ strain, the diameter in local region close to the middle of the nanowire is obviously smaller than that of two ends. This means that the tensile strain causes some area constriction of local region, resulting in the formation of the neck. With further increase in strain, the diameter of the cross-section area of the neck becomes smaller and smaller; finally a point is reached where the narrowest diameter is zero at $39.40 \%$ strain, and the nanowire breaks (see Fig. 5). Comparing the diameter distribution at three different strains $(0,11.8 \%$, and $39.4 \%)$, we find that there is only a little increase in the length of the region beyond the neck, for instance, it is 23.63 and 24.66 for $11.8 \%$ and $39.4 \%$, respectively; but the length of neck region has a significant increase from 20.33 to 30.14 . This indicates that after the formation of the neck, the plastic deformations have been carried mainly through the elongation of the neck region; the reason is that after the critical strain (about $11.8 \%$ ), further plastic deformation is localized in the neck region, since the stress increases continually with areal contraction even through the calculated stress at two ends of the nanowire is decreasing. Beyond the region of the neck, the atomic structures have no significant change through CAN methods.

As described in the previous section, when the strain is lower than $11 \%$, the deformation is in the elastic stage. As the stress is increased up to the critical level with the increase in strain, plastic

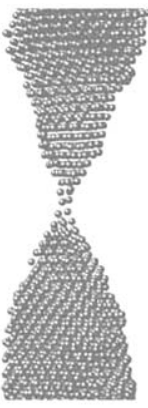

(a)

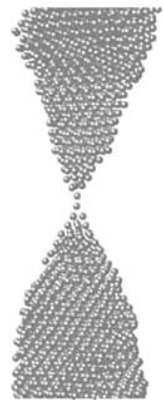

(b)

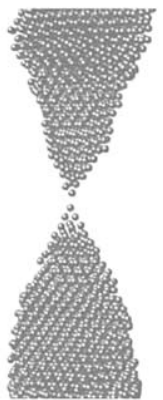

(c)

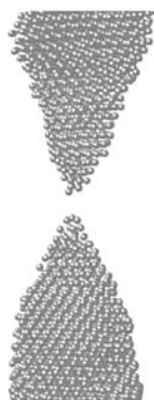

(d)
Fig. 5. Snapshots of atomic configurations of Ni nanowire at four different strains from $38.64 \%$ to $39.44 \%$, deformed at $300 \mathrm{~K}$ and the strain rate of $0.1 \% / \mathrm{ps}$. The configurations presented correspond to the following strain: (a) $38.64 \%$; (b) $39.12 \%$; (c) $39.40 \%$; (d) $39.44 \%$. 
deformation occurs. The stress-strain curve, as shown in Fig. 1, gives a zigzag increase-decrease in stress when the strain is increased to above $11.8 \%$. Comparing Figs. 1 and 2, it can be seen that the atomic structures vary with the cycle of increasedecrease in stress. It means that the atomic rearrangements happen in the system, and these rearrangements induce decrease of the stress. Noticeably, the first rearrangement, mainly the slips in the $\left\{\begin{array}{lll}1 & 1 & 1\end{array}\right\}$ planes, induces large changes of the atomic structures. Subsequently, the series of other rearrangements do not induce significant change in the nanowire. These successive rearrangements happen in the region of the neck. With the increased strain, the range of increase-decrease in stress reduces little by little, and finally the breaking of the nanowire occurs at the strain of $39.4 \%$.

In Fig. 5 we present snapshots (Figs. 5a-d) of atomic configurations before and after the failure, obtained for the nanowire at the strain between $38.64 \%$ and $39.44 \%$. As the nanowire is pulled, the diameter of the neck tends to become smaller and smaller; when the strain is increased up to $38.64 \%$, the cross-section of the neck includes only two atoms, as displayed in Fig. 5(a). With the strain up to $39.12 \%$, those atoms at the 'tip' of the neck rearrange themselves into an one-atom thick, about three-atoms long necklace (see Fig. 5(b)) which is some similar to what happened in the gold nanowire [18,21]. However, this is not observed for $\mathrm{Ni}$ nanowire simulated by Sorensen et al. [17]. With the further pull of the nanowire, the bond between the atoms in the necklace breaks, as shown in Fig. 5(c), and then rupture happens. Once the breaking happened, the atoms around the 'tips' shrink rapidly (presented in Fig. 5(d)), and correspondingly the stress decreases to around zero. The atomic structures near the 'tips' appear much highly disordered.

\section{Conclusions}

In conclusion, we have used molecular dynamics simulations with the quantum Sutten-Chen manybody force field to study the deformations on nickel nanowire subjected to the uniaxial tensile strain at $300 \mathrm{~K}$, as well as the structural evolution of nickel nanowire during the elongation process. Our results show that the elastic deformation can reach about $11 \%$ strain, and at elastic stage the deformation happened mainly through the homogeneous elongation of distances between the layers (perpendicular to the $Z$-axis) while the atomic structure remains basically unchanged. With further strain, the slips in the $\left\{\begin{array}{llll}1 & 1 & 1\end{array}\right\}$ planes happened in order to partially carry the deformation, and the plastic deformation started to take place in order to accommodate the applied strain. The neck of the nanowire forms after the slips happened, and the deformations have been carried mainly through the elongation of the neck. Beyond the neck region, atomic structures have no significant changes. The atomic rearrangements in the neck region induce the zigzag increase-decrease in stress as the strain is increased. The atoms, close to the narrowest region of the neck, are highly disordered. At the point of breaking, we observe a one-atom thick necklace. With further pulling of the nanowire, the bond between the two atoms lying in the necklace breaks and then the rupture happens. The strain rates in our simulation range have no significant effect on the deformation mechanism; the yield stress, the elastic limit, and the strain of failure increase weakly with increase in strain rate.

\section{Acknowledgements}

This work is financially supported by the National Natural Science Foundation of China (No. 10374076 and No. 10172088) and the Natural Science Foundation of Fujian Province of China (No. E0320001).

\section{References}

[1] H. Gleiter, Prog. Mater. Sci. 33 (1989) 223.

[2] H. Gleiter, Nanostruct. Mater. 6 (1995) 3.

[3] H. Gleiter, Acta Mater. 48 (2000) 1.

[4] Y. Kondo, K. Takayanagi, Science 289 (2000) 606.

[5] B.A. Glavin, Phys. Rev. Lett. 86 (2001) 4318.

[6] S. Iijima, L.C. Qin, et al., Science 296 (2002) 611.

[7] A. Bietscha, B. Michel, Appl. Phys. Lett. 80 (2002) 3346. 
[8] M. Kawamura, et al., Phys. Rev. Lett. 91 (2003) 096102.

[9] N.A. Melosh, et al., Science 300 (2003) 112.

[10] D. Srivastava, et al., in: H. S. Nalwa (Ed.), Handbook of Nanostructured Materials and Nanotechnology, vol. 2, Academic Press, New York, 2000, p. 665.

[11] D. Erts, H. Olin, J.D. Holmes, Proc. SPIE 5123 (2003) 248.

[12] G.M. Finbow, et al., Mol. Phys. 92 (1997) 705.

[13] P.S. Branicio, J.P. Rino, Phys. Rev. B 62 (2000) 16950.

[14] H. Ikeda, et al., Phys. Rev. Lett. 82 (1999) 2900.

[15] U. Landman, et al., Phys. Rev. Lett. 77 (1996) 1362.

[16] A.M. Bradkovsky, A.P. Sutton, T.N. Todorov, Phys. Rev. B 52 (1995) 5036.

[17] M.R. Sorensen, M. Brandbyge, K.W. Jacobsen, Phys. Rev. B 57 (1998) 3283.

[18] E.Z. da Silva, A.J.R. da Silva, A. Fazzio, Phys. Rev. Lett. 87 (2001) 256102.

[19] P. Jelınek, R. Perez, J. Ortega, F. Flores, Phys. Rev. B 68 (2003) 085403.

[20] A. Nakamura, et al., Phys. Rev. Lett. 82 (1999) 1538.

[21] E.Z. da Silva, et al., Phys. Rev. B 69 (2004) 115411.

[22] R.N. Barnett, U. Landman, Nature 387 (1997) 788.

[23] P. Walsh, W. Li, R.K. Kalia, A. Nakano, P. Vashishta, Appl. Phys. Lett. 78 (2001) 3328.

[24] Y. Kimura, Y. Qi, T. Cagin, W.A. Goddard, unpublished.

[25] H. Ikeda, et al., Phys. Rev. Lett. 82 (1999) 2900.

[26] Y. Qi, A. Strachan, T. Cagin, W.A. Goddard III, Mater. Sci. Eng. A 309 (2001) 156.
[27] Y. Qi, Y.T. Cheng, T. Cagin, W.A. Goddard III, Phys. Rev. B 66 (2002) 085420.

[28] D.J. Evans, B.L. Holian, J. Chem. Phys. 83 (1985) 4096.

[29] H.J.C. Berendsen, et al., J. Chem. Phys. 81 (1984) 3684.

[30] M. Amini, D. Fincham, Comput. Phys. Commun. 56 (1990) 313.

[31] J.D. Honeycutt, H.C. Andersen, J. Phys. Chem. 91 (1987) 4950.

[32] J. Schiotz, F.D.D. Tolla, K.W. Jacobsen, Nature 391 (1998) 561.

[33] V. Yamakov, D. Wolf, S.R. Phillpot, et al., Nat. Mater. 1 (2002) 1.

[34] H.B. Liu, K.Y. Chen, G.Y. An, et al., Philos. Mag. B 76 (1997) 75.

[35] E.O. Hall, Proc. Phys. Soc. Lond. B 64 (1951) 747.

[36] N.J. Petch, J. Iron Steel Inst. 174 (1953) 25.

[37] A.H. Chokshi, A. Rosen, J. Karch, H. Gleiter, Scripta Mater. 23 (1989) 1679.

[38] J.R. Weertman, Mater. Sci. Eng. A 166 (1993) 161.

[39] S. Takeuchi, Scripta Mater. 44 (2001) 1483.

[40] Z. Budrovic, H.V. Swygenhoven, et al., Science 304 (2004) 273.

[41] Y. Qi, T. Cagin, W.L. Johnson, W.A. Goddard, J. Chem. Phys. 115 (2001) 385.

[42] R.W. Hertzberg, Deformation and Fracture Mechanics of Engineering Materials, Wiley, New York, 1983, p. 6. 